an komplizierten Anträgen oft die einzige Möglichkeit darin besteht, arbeitsteilig vorzugehen und seinem Fachkollegen zu folgen. Eine sachlich begründete Ablehnung der Mehrheitsmeinung der Fraktion ist in vielen Fällen rein zeitlich für den einzelnen Abgeordneten nicht zu leisten, und diejenigen, die dennoch häufiger nicht mit der Fraktion stimmen, sind schnell innerhalb der Gruppe isoliert. Auch wenn also der Fraktionsvorstand immer noch nicht vorgibt (und in Probeabstimmungen testet), wie abgestimmt werden soll, hat sich faktisch eine Art Fraktionsdisziplin herausgebildet.

Die neuen Ideen der Piratenpartei haben also in vielen Fällen die Begegnung mit der Realität nicht überstanden. Der Druck der Öffentlichkeit, aber auch der Wunsch einiger Abgeordneter, professionellere, medientauglichere Arbeit zu leisten, hat dazu ebenso beigetragen wie schlicht der arbeitsintensive Alltag im Parlament. Da diese Gründe in Zukunft nicht abnehmen werden, sind weitere Veränderungen zu erwarten. Teile der Basis und einige Abgeordnete werden das mit Schrecken, andere mit Freude sehen. Der Fokus auf die bereits erfolgten Veränderungen sollte aber nicht den Blick verstellen auf die vielen kleinen und großen Neuerungen, die die Piratenfraktion ausprobiert und beibehalten hat - in vielerlei Hinsicht ist sie tatsächlich (noch) „anders“.

\title{
Innerparteiliche Willensbildung und Entscheidungsprozesse durch digitale Partizipation. Ein Praxistest des Konzepts der Liquid Democracy
}

\section{Bastian Bullwinkel und Lothar Probst}

Der Hype um die Piratenpartei, der noch 2011 und 2012 die Medien, aber auch die Politikwissenschaft elektrisiert hat, ist längst vorbei. In der öffentlichen Wahrnehmung spielt die Partei trotz der Enthüllungen des Whistleblowers Edward Snowden, die eigentlich den Piraten hätten Auftrieb geben müssen, kaum noch eine Rolle. Bei den letzten Landtagswahlen und bei der Bundestagswahl 2013 landeten sie weit abgeschlagen unterhalb der Fünf-Prozent-Hürde. Trotz dieser ernüchternden Bilanz lohnt es sich, die wissenschaftliche Auseinandersetzung mit der Piratenpartei und ihrem Versprechen auf umfassende Mitgliederdemokratie fortzusetzen. Es ist schwerlich zu bestreiten, dass sie einen nachhaltigen Effekt auf die anderen Parteien und deren Formen der Mitgliederbeteiligung ausgeübt hat. Selten zuvor gab es so viele Angebote an die Mitglieder der Parteien, sich aktiv an Entscheidungsprozessen zu beteiligen. Viele Instrumente sind nicht neu und wurden auch in der Vergangenheit schon in der einen oder anderen Situation ausprobiert, aber die Beispiele sind zahlreicher geworden und zeugen von einer neuen Qualität. Sie reichen von der Urwahl der Spitzenkandidaten bei den Grünen über den Mitgliederentscheid bei der Auswahl von Programmschwerpunkten bis hin zur Mitgliederbefragung der SPD über den mit den Unionsparteien ausgehandelten Koalitionsvertrag - ein Novum in der Geschichte der Regierungsbildung, jedenfalls auf Bundesebene. ${ }^{1}$ Doch auch wenn die anderen Parteien in

1 Zu direkter Demokratie in Parteien vgl. auch den Beitrag von Rudolf Steinberg in diesem Heft der ZParl. 
puncto Mitgliederbeteiligung nachgezogen haben, behalten die Piraten das Urheberrecht für das Konzept der Liquid Democracy und das dafür verwendete Instrument des LiquidFeedback. Wie sich Anspruch und Wirklichkeit bei der Realisierung von Liquid Democracy verhalten und ob sich mithilfe des LiquidFeedback eine neue Qualität der Mitgliederpartizipation erreichen lässt, soll anhand von jeweils zwei Fallbeispielen aus den Landesverbänden und Fraktionen der Piratenpartei in Berlin und Nordrhein-Westfalen untersucht werden.

\section{Untersuchungsrahmen und Methodik}

Im September 2011 zogen 15 Piraten nach einem Wahlergebnis von 8,9 Prozent in das Berliner Abgeordnetenhaus ein. Weitere Erfolge schlossen sich bei den Landtagswahlen 2012 an: 7,4 Prozent im Saarland, 8,2 Prozent in Schleswig-Holstein und 7,8 Prozent bei der Landtagswahl in Nordrhein-Westfalen. Dabei wurde die Partei vor allem über ihr Selbstverständnis wahrgenommen, Politik „neu“ und „anders“ gestalten zu wollen. Dies sollte durch eine direkte und kontinuierliche Beteiligung der Mitglieder an Entscheidungen in Form einer „flüssigen Demokratie“ geschehen - der so genannten Liquid Democracy, einer Kombination aus direkter und repräsentativer Demokratie. Um zu untersuchen, wie das Konzept der Liquid Democracy die innerparteilichen Beteiligungs- und Entscheidungsprozesse der Piratenpartei auf Landes- und Fraktionsebene strukturiert, wurden zwei Beispiele ausgewählt, und zwar Berlin und Nordrhein-Westfalen. ${ }^{2}$ Von den vier Landesverbänden mit parlamentarischer Vertretung sind diese beiden nicht nur die mitgliederstärksten, sondern sie stellen in diesen Landesparlamenten auch ihre größten Fraktionen. Zudem nutzen beide Landesverbände beziehungsweise Fraktionen die Internet-Plattform LiquidFeedback, um Meinungsbilder der Parteibasis einzuholen, die in die Entscheidungen einfließen sollen. Für beide Bundesländer werden zwei Fallbeispiele untersucht, jeweils eines auf Landesverbands- und Fraktionsebene. Für Berlin handelt es sich dabei um die Diskussion einer Pflichtangabe des bürgerlichen Namens - der Klarnamenpflicht - bei innerparteilichen Abstimmungen im Online-Tool LiquidFeedback sowie um eine Kontroverse über die Unterstützung einer gesetzlichen Frauenquote durch die Unterzeichnung der so genannten Berliner Erklärung. Im Landesverband Nordrhein-Westfalen geht es um die Positionierung in den Debatten um religiöse Beschneidungen und um die Novellierung des Nichtraucherschutzgesetzes. Die Untersuchung der Fallbeispiele hat deskriptiven Charakter und ist qualitativ ausgerichtet. Sie bedient sich einer Dokumentenanalyse, die durch Experteninterviews ergänzt wird. Der Vorteil der Dokumentenanalyse besteht darin, „dass die Daten im natürlichen Kontext entstanden sind und robuste, non-reaktive Informationen darstellen "3. Sie reagieren somit nicht auf nachfolgende Ereignisse, sondern sind Ausdruck der Zeit, in der sie entstanden sind. Die Quellen werden von Anfang an selektiv betrachtet, um nur die nötigen Informationen und Daten aus ihnen herauszufiltern. In der vorliegenden Untersuchung liegt der Fokus auf den Abstimmungen der Parteimitglieder auf der Online-Plattform LiquidFeedback. Des Weiteren wurden Satzungen der Piratenpartei, Pro-

2 Zur Piratenfraktion nach zwei Jahren im Berliner Abgeordnetenhaus vgl. auch den Beitrag von Carsten Koschmieder in diesem Heft der ZParl.

3 Detlef Jahn, Einführung in die vergleichende Politikwissenschaft, Wiesbaden 2006, S. 200. 
tokolle und Beschlüsse von Parteitagen, Positionspapiere und Pressemitteilungen, Gesetzentwürfe, aber auch - bedingt durch die modernen Kommunikationsformen der Piratenpartei - Einträge im Piratenwiki (der Online-Enzyklopädie der Piraten) sowie Blogeinträge von Parteimitgliedern einbezogen.

Zusätzlich wurden leitfadengestützte Experteninterviews durchgeführt, um die aus der Dokumentenanalyse gewonnenen Erkenntnisse anzureichern und zu ergänzen. ${ }^{4}$ Bei solchen Interviews gilt es vorab zu klären, welche Personen als Experten definiert werden und welche Experten für das individuelle Forschungsvorhaben in Frage kommen. Für die vorliegende Analyse war es sinnvoll, auf die Sachkunde von Personen zuzugreifen, die hohe Funktionen innerhalb der Piratenpartei in Berlin und Nordrhein-Westfalen bekleiden und somit nah an den Entscheidungsprozessen sind. Auf Grundlage dieser Kriterien wurden die jeweiligen Landes- und Fraktionsvorsitzenden der Piratenpartei aus Berlin und NordrheinWestfalen ausgewählt. ${ }^{5}$

Die Untersuchung hat aufgrund der verwendeten qualitativen Methoden in erster Linie explorativen Charakter und lässt aufgrund der begrenzten Anzahl von Fallbeispielen keine verallgemeinerungsfähigen Aussagen zu. Gleichwohl werden zwei Annahmen aufgestellt, die im Verlauf der Studie überprüft werden sollen:

(1) Durch die niedrigschwelligen Hürden digitaler Partizipation beteiligen sich mehr Mitglieder der Piratenpartei an internetbasierten innerparteilichen Entscheidungsprozessen, als es auf Parteitagen der Fall ist.

(2) Durch die Anwendung von Liquid Democracy binden sich die Landes- und Fraktionsvorsitzenden der Piratenpartei an die Meinungsbilder der Parteibasis.

Zur Überprüfung der ersten Annahme werden die Beteiligungsraten ausgewählter Entscheidungsprozesse im Internet mit den Teilnehmerzahlen der Landesparteitage der Piraten in Berlin und Nordrhein-Westfalen verglichen. Beteiligen sich mehr Parteimitglieder an den internetbasierten Entscheidungen als an den Entscheidungen auf den Landesparteitagen, so ist dies ein Hinweis auf die Richtigkeit der Annahme. Die zweite Annahme wird anhand der Entscheidungsprozesse in den ausgewählten Fallbeispielen überprüft. Sollten sich Landesvorstand und Parlamentsfraktion jeweils an das Meinungsbild der Parteibasis halten, spricht dies für die Richtigkeit der zweiten Annahme. In beiden Fällen verbietet sich aber aus den genannten Gründen die Verallgemeinerung.

\section{Entstehung und Entwicklung des Konzepts der Liquid Democracy}

Das Konzept der Liquid Democracy erhebt den Anspruch, für eine neue Form der Demokratie zu stehen und bestehende Erstarrungen und Begrenzungen demokratischer Systeme

4 Vgl. Michael Meuser / Ulrike Nagel, Das Experteninterview - konzeptionelle Grundlagen und methodische Anlage, in: Susanne Pickel / Gert Pickel / Hans-Joachim Lauth / Detlef Jahn (Hrsg.), Methoden der vergleichenden Politik- und Sozialwissenschaft. Neue Entwicklungen und Anwendungen, Wiesbaden 2009, S. 465 - 479.

5 Mit folgenden Vertretern der Piratenpartei wurden im November und Dezember 2012 Experteninterviews durchgeführt: Gerhard Anger (Landesvorsitzender Berlin), Andreas Baum (Fraktionsvorsitzender Berlin), Sven Sladek (Landesvorsitzender Nordrhein-Westfalen) und Joachim Paul (Fraktionsvorsitzender Nordrhein-Westfalen). 
aufzuweichen und zu verflüssigen. ${ }^{6}$ Jeder Bürger $^{7}$ beziehungsweise jedes Parteimitglied kann hierbei zu jeder Zeit an politischen Entscheidungsprozessen teilnehmen. Liquid Democracy wird heutzutage zwar vor allem mit der Piratenpartei in Verbindung gebracht, der dahinter stehende Grundgedanke existiert jedoch schon länger.

\subsection{James C. Miller-Direct and Proxy Voting}

Die ersten theoretischen Ansätze einer „flüssigen Demokratie“ lieferte der amerikanische Wirtschaftswissenschaftler James C. Miller Mitte des 20. Jahrhunderts. Für ihn stellte sich die Frage, warum auf nationaler Ebene repräsentative Entscheidungsprozesse den direkten vorgezogen werden, anstatt sie miteinander zu verbinden. Vor dem Hintergrund der für ihn offensichtlichen Nachteile einer direkten Demokratie - (1) örtliche Einschränkungen, (2) nur wenige Personen fühlen sich kompetent genug, um über eine Bandbreite von Themen zu entscheiden sowie (3) ein Mangel an Zeit, sich in alle Themen einzuarbeiten - erstellte er ein Programm, das die positiven Eigenschaften der direkten Demokratie mit der Notwendigkeit der repräsentativen Demokratie verknüpfen sollte. ${ }^{8}$

Statt Abgeordnete in festgesetzten Wahlperioden zu wählen, schlug Miller vor, jeden Bürger zu allen politischen Fragen selbst abstimmen zu lassen oder aber ihm die Möglichkeit zu geben, seine Stimme an einen Stellvertreter - einen so genannten proxy - zu delegieren. Diese Delegation sollte jederzeit widerrufbar sein. Dadurch würden sich drei Kategorien von Wählern entwickeln: diejenigen, die über jeden Sachverhalt selbst abstimmen; diejenigen, die zu jedem Thema ihre Stimme delegieren sowie diejenigen, die beide Extreme miteinander verbinden und je nach Thema eigenständig abstimmen oder ihren proxy wählen lassen. ${ }^{9}$

Das vorgeschlagene System, so Miller, biete Vorzüge gegenüber rein repräsentativen Formen. Unter anderem bekäme dadurch im Endergebnis jede einzelne Stimme mehr Gewicht. ${ }^{10}$ Die Möglichkeit der temporären Stimmübertragung löse zudem das Problem, dass nicht jeder Bürger und auch nicht jeder Abgeordnete Experte in jedem Politikfeld sein könne. Fühle man sich in einem bestimmten Thema nicht kompetent genug, könne man seine Stimme einfach an jemanden übertragen, dem man die nötige Expertise zuspricht. Gleichzeitig weiche das System auch die räumliche Begrenzung auf, indem die Repräsentanten nicht aus demselben Gebiet stammen müssen wie die Person, in deren Namen sie abstimmen. Durch die Wahl eines proxy können Vertreter aus der ganzen Nation gewählt werden, unabhängig davon, ob sie in derselben Region wie der jeweilige Bürger wohnen. ${ }^{11}$

6 Vgl. Sebastian Buck, Liquid Democracy - eine Realisierung deliberativer Hoffnungen? Zum Selbstverständnis der Piratenpartei, in: ZParl, 43. Jg. (2012), H. 3, S. 626 - 635.

7 Aus Gründen der besseren Lesbarkeit wird auf die gleichzeitige Verwendung männlicher und weiblicher Sprachformen verzichtet. Sämtliche Personenbezeichnungen in diesem Beitrag gelten für beiderlei Geschlecht.

8 Vgl. James $C$. Miller, A program for direct and proxy voting in the legislative process, in: Public Choice, 7. Jg. (1969), H. 1, S. $107-113$.

9 Vgl. ebenda, S. 108.

10 Vgl. ebenda, S. 109 f.

11 Vgl. ebenda, S. 111. 


\subsection{Bryan Ford - Delegative Democracy}

$\mathrm{Zu}$ Beginn des 21. Jahrhunderts wurde der Entwurf von James C. Miller erneut aufgegriffen. Bryan Ford, Professor an der Universität in Yale, führte ihn fort und entwickelte den Begriff der Delegative Democracy. Dieses Konzept kombiniert dem Autor zufolge die besten Elemente direkter und repräsentativer Demokratie und hebt dabei individuelle Stimmendelegationen gegenüber „mass election“ "hervor. ${ }^{12}$ Sein Entwurf sieht vor, dass die Wähler die größtmögliche direkte Wahl ihrer Repräsentanten haben. Diese werden als delegates bezeichnet und nicht mehr, wie im Entwurf von Miller, als proxies. Zusammenfassend besteht die Delegative Democracy aus den folgenden Kernelementen ${ }^{13}$ :

(1) Jedem Mitglied der Gesellschaft steht es frei, sich aktiv durch eine Rolle als delegate oder passiv durch Stimmdelegation in die Entscheidungsprozesse einzubringen. Delegierte haben außerdem die Wahl, wie aktiv sie sein möchten und in welchen Themenbereichen.

(2) Die Anzahl der Delegierten sollte nicht beschränkt sein. Um sicherzustellen, dass es jeder Delegierte ernst meint, kommen jedoch leichte Hürden infrage, wie zum Beispiel ein gewisses Verständnis für die Strukturen und Abläufe der jeweiligen Institution.

(3) Delegierte agieren nur für sich selbst sowie im Namen derer, die ihre Stimme an sie übertragen haben. Das Stimmgewicht der einzelnen Delegierten variiert dabei je nachdem, wie viele Stimmen dem Delegierten übertragen wurden.

(4) Um sozialen Druck zu minimieren, wird das Stimmverhalten der einzelnen Wähler geheim gehalten. Den Delegierten soll es nicht möglich sein, zu wissen, in wessen Namen sie abstimmen.

(5) Die Stimmen der Delegierten sollen hingegen öffentlich sein. Dies biete den Delegierenden Kontrollmöglichkeiten, ob auch wirklich in ihrem Sinne abgestimmt wurde.

(6) Die oberste Regel aller Delegierten müsse sein, als Generalisten zu handeln. In Bereichen, in denen ihnen das entsprechende Wissen fehlt, können sie ihre Stimmen an Personen übertragen, die in diesem Bereich spezialisiert sind.

\subsection{Liquid Democracy durch digitale Partizipation}

Die Kernelemente von Bryan Fords Delegative Democracy bilden die Grundlage für das Konzept der Liquid Democracy, wie sie jetzt mithilfe des Internets umgesetzt werden soll. Dem Internet wird bereits seit mehreren Jahren attestiert, dass es „innerparteiliche Informationsprozesse erweitern sowie beschleunigen und infolgedessen neue Quellen des Informationszugangs und der Meinungsbildung erschließen"14 kann. Dadurch eignet es sich nach Auffassung der Piratenpartei als Grundlage für die Verwirklichung von Liquid Democracy

12 Vgl. Bryan Ford, Delegative Democracy, 15. Mai 2002, S. 1, http://www.brynosaurus.com/ $\log / 2002 / 0515-$ DelegativeDemocracy.pdf (Abruf am 4. Januar 2014).

13 Vgl. ebenda, S. $4 \mathrm{ff}$.

14 Elmar Wiesendahl, Parteienkommunikation parochial. Hindernisse beim Übergang in das Online-Parteienzeitalter, in: Ulrich von Alemann / Stefan Marschall (Hrsg.), Parteien in der Mediendemokratie, Wiesbaden 2002, S. 364 - 389, S. 380. 
sowohl in der Politik als auch in innerparteilichen Entscheidungsprozessen. ${ }^{15}$ Mithilfe von Online-Plattformen wie LiquidFeedback oder adhocracy ${ }^{16}$ könne jedes Parteimitglied oder sogar jeder wahlberechtigte Bürger an allen Willensbildungs- und Entscheidungsprozessen teilnehmen.

An der Entwicklung dieses neuartigen Demokratiekonzeptes maßgeblich beteiligt ist der Verein Liquid Democracy ${ }^{17}$, der an Ideen und Projekten zur demokratischen Teilhabe arbeitet. Wie bereits einleitend erwähnt, soll eine Liquid Democracy Erstarrungen und Begrenzungen demokratischer Systeme aufweichen. Zu diesen starren Strukturen zählt der Verein vor allem (1) die zeitliche Begrenzung von Partizipationsmöglichkeiten der Bürger - was sich zum Beispiel darin äußere, dass nur alle vier oder fünf Jahre über die Besetzung von Parlamenten entschieden werden kann; (2) die inhaltliche Begrenzung von Entscheidungen durch die Auswahlmöglichkeiten von wenigen Komplettpaketen (Parteiprogrammen) sowie (3) eine partizipatorische Begrenzung zugunsten einer politischen Elite. ${ }^{18} \mathrm{Li}$ quid Democracy zielt dagegen darauf ab, dass sich jeder Beteiligte zu jeder Zeit an allen Entscheidungsprozessen beteiligen kann. Jedoch muss er dies nicht immer, denn es gibt die Möglichkeit der Stimmdelegation, das so genannte delegated voting. Durch die Kumulation der ihm übertragenen Stimmen bekommt der Delegierte ein höheres Stimmgewicht. Alternativ kann er seine eigene und die übertragenen Stimmen ebenfalls delegieren, wodurch eine weitere Stimmenakkumulation entsteht. Die übertragenen Stimmen betreffen nur jene Themen, zu denen die Delegation stattgefunden hat. Jemand, der seine Stimme für Thema X abgegeben hat, kann also jederzeit noch selbst über Thema Y abstimmen. Auch hat er jederzeit die Möglichkeit, seine übertragene Stimme zu widerrufen, sei es, weil er nach einer gewissen Zeit doch wieder selbst über das Thema abstimmen möchte oder aber die Meinung des Delegierten nicht mehr seiner eigenen entspricht und er seine Stimme an eine andere Person übertragen möchte. ${ }^{19}$

\section{Die Piratenpartei in Deutschland}

Gegründet wurde der deutsche Ableger der schwedischen Piratpartiet am 10. September 2006 in Berlin. Mediale (und auch politikwissenschaftliche) Aufmerksamkeit erlangte die Piratenpartei in Deutschland jedoch erstmals mit ihrem Ergebnis von 0,9 Prozent bei der

15 Im Jahr 2011 waren fast 92 Prozent der Mitglieder der Piratenpartei der Meinung, dass das Internet ein Schlüsselfaktor für eine erfolgreiche Programmarbeit sei; vgl. Tobias Neumann, Die Piratenpartei in Deutschland. Entwicklung und Selbstverständnis, Berlin 2011, S. 129.

16 Adhocracy ist - wie LiquidFeedback - eine Software zur Umsetzung von Liquid Democracy und setzt somit ebenfalls auf Online-Beteiligung. Genutzt wurde das Programm unter anderem von der Enquete-Kommission „Internet und Gesellschaft“ des Deutschen Bundestages.

17 Liquid Democracy e.V. ist ein gemeinnütziger und überparteilicher Verein, der an Ideen und Projekten zur demokratischen Teilhabe arbeitet, um Entscheidungsprozesse transparenter und flexibler zu gestalten. Er wurde 2009 gegründet und hat seinen Sitz in Berlin. Der Verein ist verantwortlich für die Entwicklung der internetbasierten Open Outsource-Beteiligungssoftware Adhocracy (adhocracy.de) und betreibt weitere Beteiligungsplattformen.

18 Vgl. Liquid Democracy e.V., Liquid Democracy, https://liqd.net/schwerpunkte/theoretischegrundlagen/liquid-democracy/ (Abruf am 4. Januar 2014).

19 Vgl. Stefan Appelius / Armin Fuhrer, Das Betriebssystem erneuern. Alles über die Piratenpartei, Berlin 2011, S. 147. 
Wahl zum Europäischen Parlament im Juni 2009. Die historische Entwicklung ${ }^{20}$ und inhaltliche Ausrichtung ${ }^{21}$ der Partei sollen jedoch nicht Gegenstand dieser Untersuchung sein. Vielmehr geht es um die innerparteilichen Entscheidungsstrukturen und -prozesse.

\subsection{Organisation und Struktur der Piratenpartei}

Wie andere Parteien auch, weisen die Piraten eine klassische Gebietsgliederung in Bundes-, Landes-, Kreis- sowie Orts- beziehungsweise Bezirksverbände auf und stellen rechtlich vorgeschriebene Parteiorgane. Zu den Organen der Bundespartei zählen gemäß $₫ 9$ der Bundessatzung $^{22}$ der Vorstand, der Bundesparteitag, das Bundesschiedsgericht sowie die Gründungsversammlung. Die zweite Organisationsebene wird in $\$ 7$ der Bundessatzung festgelegt, wonach sich die Piratenpartei unterhalb der Bundespartei in 16 Landesverbände gliedert - einem pro Bundesland. Jeder Landesverband hat einen eigenen Vorstand sowie einen Landesparteitag beziehungsweise eine Landesmitgliederversammlung. Die weitere Untergliederung der Landesverbände erfolgt in Kreis- und Bezirksverbände, die mit den politischen Grenzen der Kreise, kreisfreien Städte und Gemeinden sowie Regierungsbezirken deckungsgleich sind.

Eine Besonderheit in der Organisationsstruktur der Piratenpartei sind die zahlreichen informellen Zusammenschlüsse. Dazu gehören die Arbeitsgemeinschaften, die Squads sowie die Crews. Erstere sind an spezifische Themen gebunden und existieren sowohl in der Bundespartei als auch in den Landesverbänden. ${ }^{23} \mathrm{Im}$ Berliner Landesverband lautet die Bezeichnung für die Arbeitsgemeinschaften Squad. ${ }^{24}$ Demgegenüber stehen die so genannten Crews. Dies sind Gruppen von fünf bis neun Parteimitgliedern und dienen vor allem der sozialen Vernetzung. Im Gegensatz zu den Arbeitsgemeinschaften beziehungsweise Squads sind die Crews nicht thematisch gebunden, sondern ortsbezogen. In Berlin ersetzen sie außerdem die Bezirksverbände. ${ }^{25}$

\subsection{Instrumente der innerparteilichen Entscheidungsfindung}

Zentral für die Arbeit der Piratenpartei und die Umsetzung einer Liquid Democracy sind die Instrumente der innerparteilichen Willensbildung und Entscheidungsfindung. Sowohl

20 Vgl. zur Entwicklung der Piratenpartei Deutschland unter anderem Oskar Niedermayer, Erfolgsbedingungen neuer Parteien im Parteiensystem am Beispiel der Piratenpartei Deutschland, in: ZParl, 41. Jg. (2010), H. 4, S. 838 - 854; Christoph Bieber, Die Piratenpartei als neue Akteurin im Parteiensystem, in: APuZ, 62. Jg. (2012), H. 7, S. 27 - 33.

$21 \mathrm{Vgl}$. zur thematischen Ausrichtung der Piratenpartei Deutschland unter anderem Henning Bartels, Die Piratenpartei. Entstehung, Forderung und Perspektiven der Bewegung, Berlin 2009, S. $131 \mathrm{ff}$.

22 Vgl. Piratenpartei Deutschland, Die Satzung der Piratenpartei Deutschland. Bundessatzung der Piraten, Fassung vom 5. Januar 2014, https:/www.piratenpartei.de/wp-content/uploads/ 2014/01/Bundessatzung.pdf (Abruf am 21. Januar 2014).

$23 \mathrm{Vgl}$. Piratenwiki, Arbeitsgemeinschaften, http://wiki.piratenpartei.de/Arbeitsgemeinschaften (Abruf am 21. Mai 2014).

24 Vgl. dass., Squads, http://wiki.piratenpartei.de/BE:Squads (Abruf am 21. Mai 2014).

25 Vgl. dass., Crews, http://wiki.piratenpartei.de/Crews (Abruf am 21. Mai 2014). 
Bundes- als auch Landesparteitage finden laut den entsprechenden Satzungen mindestens einmal pro Jahr auf Einladung des Vorstands statt. Ein außerordentlicher Parteitag wird nur dann einberufen, wenn ein neuer Vorstand gewählt werden muss. Im Unterschied zu den Parteitagen der meisten anderen Parteien handelt es sich bei den Piraten jedoch um keine Delegiertenversammlungen, sondern um tatsächliche Mitgliederversammlungen, zu denen prinzipiell jedes (zahlende) Parteimitglied erscheinen kann und auch stimmberechtigt ist. ${ }^{26}$

Daneben setzt die Partei vor allem auf Partizipationsplattformen im Internet. Die Software LiquidFeedback ist dabei das zentrale Werkzeug, das sowohl auf Bundesebene ${ }^{27}$ als auch auf Ebene der einzelnen Landesverbände ${ }^{28}$ eingesetzt wird. Es fungiert als Antrags-, Diskussions- und Abstimmungssystem, über das sich theoretisch jedes Mitglied beteiligen und einbringen kann. ${ }^{29}$ Die Teilnahme am LiquidFeedback ist nur Parteimitgliedern vorbehalten. Nicht-Parteimitglieder haben jedoch Leserechte auf der Plattform und können Diskussionen und Entscheidungsprozesse somit verfolgen und nachvollziehen. ${ }^{30}$

Die Oberfläche der Software gliedert sich zunächst in mehrere Themenbereiche, die in der Regel einem Politikfeld zugeordnet sind. Neben klassischen Politikfeldern wie zum Beispiel „Wirtschaft und Soziales“, „Bildung und Forschung“ oder „Umwelt, Gesundheit und Ernährung" finden sich auch Themenbereiche, die sich mit innerparteilichen Diskussionen beschäftigen. Ein Themenbereich vereinigt verschiedene Themen zur jeweiligen inhaltlichen Kategorie, jedes einzelne Thema ist wiederum einem spezifischen Anliegen innerhalb des Themenbereichs gewidmet und beinhaltet eine oder mehrere Initiativen. Jedes neue Thema durchläuft mit allen Initiativen gemeinsam verschiedene Phasen auf dem Weg von der Erstellung bis zum endgültigen Abstimmungsergebnis. Die Länge der einzelnen Phasen ist unterschiedlich und richtet sich nach dem Regelwerk beziehungsweise dem Antragsverfahren, das dem jeweiligen Thema zugrunde liegt (vgl. Tabelle 1).

In der Phase „Neu“ müssen zunächst genügend Unterstützer für ein Thema beziehungsweise die bereits erstellten Initiativen gewonnen werden. Um in die nächste Phase überzugehen, benötigt ein Thema ein Quorum von mindestens zehn Prozent. ${ }^{31}$ Gelingt dies nicht, wird es abgebrochen. Wird das Quorum erreicht, folgt eine längere Diskussionsphase, in der sich Befürworter und Gegner der einzelnen Initiativen austauschen und ihre Positionen begründen können. Die Initiativen können in dieser Phase zu jeder Zeit von ihren Autoren

26 Jedes Mitglied der Piratenpartei hat das gleiche Stimmrecht, das jedoch nur ausgeübt werden kann, wenn der erste Mitgliedsbeitrag nach Eintritt in die Partei bezahlt wurde und das Mitglied nicht mehr als drei Monate mit seinen Beiträgen im Rückstand ist. Auf Parteitagen kann das Stimmrecht nur genutzt werden, wenn alle Mitgliedsbeiträge beglichen sind. Nicht bezahlte Beiträge haben also keinen Ausschluss aus der Partei zur Folge, die Mitglieder verlieren jedoch ihr Stimmrecht.

27 Das LiquidFeedback-System der Bundespartei findet sich unter https://lqfb.piratenpartei.de/ (Abruf am 21. Mai 2014).

28 Die LiquidFeedback-Systeme der einzelnen Landesverbände finden sich unter https://qpp.de/ (Abruf am 21. Mai 2014).

29 Sofern nicht anders angegeben, beziehen sich die Ausführungen über LiquidFeedback auf den parteiinternen Eintrag über das System im Piratenwiki unter http://wiki.piratenpartei.de/LiquidFeedback (Abruf am 21. Mai 2014).

30 Von diesem Gastzugriff haben die Autoren Gebrauch gemacht, um Einblicke in die später behandelten Fallbeispiele zu erhalten.

31 Das Quorum bezieht sich nicht auf die Gesamtheit der Parteimitglieder beziehungsweise LiquidFeedback-Nutzer, sondern nur auf diejenigen, die sich als Interessent für den jeweiligen Themenbereich eingetragen haben. 


\begin{tabular}{|l|c|c|c|c|c|}
\hline \multirow{2}{*}{ Tabelle 1: Übersicht der Regelwerke im LiquidFeedback } & \multirow{2}{*}{$\begin{array}{c}\text { benötigte } \\
\text { Mehrheit }\end{array}$} \\
\cline { 2 - 5 } Regelwerk & Neu & Diskussion & Eingefroren & Abstimmung & 2/3-Mehrheit \\
\hline Satzungsänderung & 15 Tage & 30 Tage & 15 Tage & 15 Tage & 2/3-Mehrheit \\
\hline Programmantrag & 15 Tage & 30 Tage & 15 Tage & 15 Tage & einfache Mehrheit \\
\hline $\begin{array}{l}\text { sonstiger } \\
\text { Parteitagsbeschluss }\end{array}$ & 8 Tage & 15 Tage & 8 Tage & 8 Tage & einfache Mehrheit \\
\hline $\begin{array}{l}\text { Meinungsbild / } \\
\text { Beschlussvorlage }\end{array}$ & 8 Tage & 15 Tage & 8 Tage & 8 Tage & 2/3-Mehrheit \\
\hline Schnellverfahren & 30 Stunden & 30 Stunden & 30 Stunden & 60 Stunden & 2/3-Mehrheit \\
\hline $\begin{array}{l}\text { Änderung von Themenbe- } \\
\text { reichen / Quoren / Fristen }\end{array}$ & 8 Tage & 15 Tage & 8 Tage & 8 Tage & einfache Mehrheit \\
\hline Abstimmungsstreitfrage & 8 Tage & 15 Tage & 8 Tage & 8 Tage & enten \\
\hline Superschnellverfahren & 30 Minuten & 30 Minuten & 30 Minuten & 60 Minuten & einfache Mehrheit \\
\hline $\begin{array}{l}\text { Veröffentlichung } \\
30 \text { Stunden }\end{array}$ & 30 Stunden & 30 Stunden & 60 Stunden & 2/3-Mehrheit \\
\hline $\begin{array}{l}\text { Quelle: Eigene Darstellung auf Basis von Angaben unter http://wiki.piratenpartei.de/LiquidFeedback/ } \\
\text { Regelwerke (Abruf am 21. Mai 2014). }\end{array}$ \\
\hline
\end{tabular}

geändert und angepasst werden. In der nächsten Phase wird das Thema „eingefroren“, es sind keine weiteren Änderungen in den bestehenden Formulierungen möglich. Jedoch können noch weitere Initiativen hinzugefügt werden, die ihrerseits vor Ablauf der „Eingefroren“-Phase ein Quorum von mindestens zehn Prozent benötigen. Alle Initiativen, die diese Hürde überspringen, werden daraufhin zur endgültigen Abstimmung gestellt. Dabei wird eine Präferenzwahl angewandt, die nach der so genannten Schulze-Methode ausgezählt wird: Jeder Teilnehmer kann den einzelnen Initiativen zustimmen, sie ablehnen oder sich enthalten. Zusätzlich kann er noch eine Reihenfolge festlegen und dadurch Präferenzen zum Ausdruck bringen. In der Auswertung werden dann alle Initiativen paarweise gegenübergestellt. Die Initiative mit der größten Zustimmung gegenüber den jeweils anderen Initiativen gewinnt, wobei auch sie die benötigte Mindestzustimmung entsprechend des Regelwerks erreichen muss. ${ }^{32}$

Wie im theoretischen Konzept der Liquid Democracy gibt es auch in der Umsetzung per LiquidFeedback die Möglichkeit, Stimmen zu delegieren. Dabei wird zwischen einer globalen Delegation für alle Themenbereiche und Themen, einer Delegation für bestimmte Themenbereiche sowie einer für einzelne Themen unterschieden. Eine Stimmübertragung lässt sich dabei ohne weiteres wieder entziehen. Stimmt ein Mitglied selbst ab, ohne vorher die Delegation zurückgezogen zu haben, zählt automatisch die eigene Stimme und die Delegation wird ausgesetzt. Ebenso hat die Übertragung für ein einzelnes Thema immer Vorrang vor einer Themenbereichsdelegation und diese wiederum vor einer globalen Delegation. Mitglieder, auf die eine oder mehrere Stimmen übertragen wurden, haben zusätzlich die Möglichkeit, diese, inklusive der eigenen, an ein weiteres Mitglied zu delegieren.

32 Vgl. Sebastian Jabbusch, Liquid Democracy in der Piratenpartei. Eine neue Chance für innerparteiliche Demokratie im 21. Jahrhundert?, Greifswald 2011, S. 63 f., http://www.sebastianjabbusch.de/wp-content/uploads/2011/10/Liquid-Democracy-in-der-Piratenpartei-Eine-NeueChance-fur-die-innerparteiliche-Demokratie-im-21-Jahrhundert-By-Sebastian-Jabbusch.pdf (Abruf am 21. Mai 2014). 
Das Piratenwiki ${ }^{33}$ ist eine Online-Enzyklopädie und wird von der Piratenpartei als Informations- und Koordinationsplattform genutzt. Auf Grund der Fülle an Informationen, die es beinhaltet, kann es auch als das „Online-Gedächtnis“ der Piraten bezeichnet werden. ${ }^{34}$ Da jeder Nutzer Seiten erstellen kann, handelt es sich nicht bei allen Einträgen um offizielle Aussagen der Partei. Ist dies jedoch der Fall - wie zum Beispiel bei Parteiprogrammen und Positionspapieren -, so sind diese Seiten mit einem Vermerk gesperrt und können nur von autorisierten Personen oder per Antrag auf dem nächsten Parteitag geändert werden. Neben den Mitgliederversammlungen, LiquidFeedback sowie dem Piratenwiki nutzt die Piratenpartei noch eine Reihe weiterer digitaler Werkzeuge, die zum Teil jedoch mehr der reinen Kommunikation und weniger als Instrumente innerparteilicher Entscheidungsprozesse dienen - zum Beispiel ein Forum, verschiedene Mailinglisten, die Besprechungssoftware Mumble sowie das Textbearbeitungstool Piratenpad.

\section{Liquid Democracy im Praxistest - die Fallbeispiele}

\subsection{Die Piratenpartei in Berlin}

Der am 30. Dezember 2006 gegründete Landesverband der Piraten in Berlin ist mit derzeit etwa 3.300 Mitgliedern ${ }^{35}$ nach den Landesverbänden Bayern und Nordrhein-Westfalen der drittgrößte. Mit einem Ergebnis von 8,9 Prozent bei der Wahl zum Berliner Abgeordnetenhaus im September 2011 gewann die Piratenpartei 15 Sitze. Die Landesmitgliederversammlung tagt mindestens einmal pro Jahr. 2012 haben im Schnitt 11,6 Prozent der Parteimitglieder daran teilgenommen (vgl. Tabelle 2), 2011 waren es nach Auskunft des Landesvorstandes immer zwischen 10 und 20 Prozent der Parteimitglieder. ${ }^{36}$

\begin{tabular}{|l|c|c|c|}
\hline Tabelle 2: Teilnehmerzahlen der Landesmitgliederversammlungen der Piratenpartei Berlin \\
\hline Landesmitgliederversammlung & Anzahl Teilnehmer & Anzahl Parteimitglieder & Anteil (in Prozent) \\
\hline 2012.2 & 403 & 3.799 & 10,6 \\
2012.1 & 340 & 2.700 & 12,6 \\
\hline Durchschnitt & - & - & 11,6 \\
\hline Anmerkung: Die Bezeichnung beziehungsweise Nummer des Parteitages richtet sich bei den Piraten \\
nach der Jahreszahl und der Anzahl der Parteitage im jeweiligen Jahr. Die zweite Landesmitgliederver- \\
sammlung der Berliner Piraten trägt daher zum Beispiel die Bezeichnung „2012.2“. \\
Quelle: Eigene Darstellung und Berechnung auf Basis von Angaben des Vorstandes der Piratenpartei \\
Deutschland Berlin.
\end{tabular}

33 Das Piratenwiki findet sich im Internet unter http://wiki.piratenpartei.de (Abruf am 21. Mai 2014).

34 Jan Odenbach, Partei, Netz, Netzpartei. Meinungs- und Willensbildung in der Piratenpartei, Wiesbaden 2012, S. 66.

35 Die Mitgliederzahlen der Piratenpartei werden regelmäßig im Piratenwiki unter http://wiki.piratenpartei.de/Mitglieder aktualisiert (Abruf am 21. Mai 2014).

36 Die Zahlen der Parteitage beziehen sich lediglich auf die Jahre 2011 und 2012, da keine offiziellen Zahlen für 2013 vorhanden sind und somit auch eine bessere Vergleichbarkeit zu den Fallbeispielen aus dem Jahr 2012 gewährleistet wird. 
(a) Landesverband Berlin: Abstimmung über eine Klarnamenpflicht im Liquid Feedback

Seit Anfang 2010 setzt der Landesverband Berlin auf die Software LiquidFeedback zur innerparteilichen Entscheidungsfindung. In der Satzung heißt es in $\$ 11$ : „Zwischen den Mitgliederversammlungen nutzt die Piratenpartei Deutschland Berlin das Konzept der Liquid Democracy zur Willensbildung über das Internet. “37 Die dabei getroffenen Entscheidungen der Parteibasis werden zwar als „Empfehlungen“ qualifiziert, sollen aber den offiziellen Parteiorganen als Grundlage ihrer Beschlüsse dienen. Eine Abweichung von den Empfehlungen ist zu begründen. Anträge aus dem Liquid Democracy-System sind vorrangig zu behandeln. Zudem sieht die Satzung der Berliner Piraten seit der Landesmitgliederversammlung im März 2014 eine ständige Mitgliederversammlung (SMV) über das Internet vor. Darüber soll zukünftig unter anderem über politische Stellungnahmen sowie Änderungen und Ergänzungen des Wahl- und des Grundsatzprogramms online abgestimmt werden.

Im Vorfeld der ersten Mitgliederversammlung des Berliner Landesverbandes im Jahr 2012 wurde im LiquidFeedback über die Einführung einer namentlichen Überprüfung der angemeldeten Nutzer diskutiert, um die Ergebnisse besser überprüfen und legitimieren zu können. Insgesamt sechs Initiativen wurden im Thema \#663, das mit dem Regelwerk „Meinungsbild / Beschlussvorlage“ angelegt wurde, unter der Rubrik „Satzung, Parteistruktur und GO [Geschäftsordnung] der LMV [Landesmitgliederversammlung] “ diskutiert und zur Abstimmung gebracht. ${ }^{38}$ Erstellt wurde das Thema am 16. Februar 2012 mit den folgenden Initiativen:

(1) In der Initiative \#1420 wird gefordert, durch eine Satzungsänderung eine Pflicht zur Angabe des bürgerlichen Namens bei Abstimmungen im LiquidFeedback einzuführen. ${ }^{39}$

(2) Initiative \#1442 bezieht keine inhaltliche Stellung zum Thema, argumentiert jedoch, dass das Thema unter einem falschen Regelwerk eingestellt worden sei und für eine Annahme des Vorschlags eine Zwei-Drittel-Mehrheit nötig sei und nicht nur eine einfache Mehrheit. ${ }^{40}$

(3) In der Initiative \#1470 wird die Forderung nach einer Klarnamenpflicht inhaltlich unterstützt, jedoch mit sprachlichen Veränderungen im Gegensatz zur Ursprungsinitiative \#1420. ${ }^{41}$

(4) Die Initiative \#1476 spricht sich für eine klarere Formulierung des Satzungsänderungsentwurfs zugunsten einer Klarnamenpflicht aus. Demnach soll künftig nicht nur der bürgerliche Name, sondern auch der Rufname plus Mitgliedsnummer jedes Teilnehmers für alle Teilnehmer im System sichtbar sein. ${ }^{42}$

(5) Die Initiative \#1477 schlägt vor, dass es den Teilnehmern selbst überlassen bleiben soll, ob ihr bürgerlicher Name oder ein Pseudonym angezeigt wird. ${ }^{43}$

37 Piratenpartei Deutschland Berlin, Satzung des Landesverbandes Berlin der Piratenpartei Deutschland, o.O. 2014, http://berlin.piratenpartei.de/partei/satzung/ (Abruf am 21. Mai 2014).

38 Vgl. LiquidFeedback der Piratenpartei Deutschland Berlin, Thema \#663, https://lqpp.de/be/issue/show/663.html (Abruf am 21. Mai 2014).

$39 \mathrm{Vgl}$. dass., Initiative \#1420, https://qpp.de/be/initiative/show/1420.html (Abruf am 21. Mai 2014).

40 Vgl. dass., Initiative \#1442, https://lqpp.de/be/initiative/show/1442.html (Abruf am 21. Mai 2014).

41 Vgl. dass., Initiative \#1470, https://qpp.de/be/initiative/show/1470.html (Abruf am 21. Mai 2014).

42 Vgl. dass., Initiative \#1476, https://qpp.de/be/initiative/show/1476.html (Abruf am 21. Mai 2014).

43 Vgl. dass., Initiative \#1477, https://qpp.de/be/initiative/show/1477.html (Abruf am 21. Mai 2014). 
(6) Eine Satzungsänderung in Form einer Klarnamenpflicht wird im Rahmen der Initiative \#1484 abgelehnt, da sie unnötig sei und den Betrieb des Liquid Democracy-Systems einschränke. ${ }^{44}$

In der anschließenden Abstimmungsphase gaben 137 Mitglieder entweder persönlich oder über eine Delegation ihre Stimme ab. Dies entspricht bei einer damaligen Mitgliederzahl von 2.700 im Berliner Landesverband einem Anteil von 5,1 Prozent. Lediglich eine Initiative erreichte die notwendige Zustimmungsrate von über 50 Prozent und zwar \#1470 mit 71 Prozent. Somit sprach sich eine deutliche Mehrheit der teilnehmenden Mitglieder für eine Satzungsänderung aus, um die Pflicht zur namentlichen Abstimmung im LiquidFeedback zu verankern.

Der Antrag wurde nach der erfolgreichen Abstimmung im LiquidFeedback auf der folgenden Landesmitgliederversammlung der Piratenpartei Berlin am 25./26. Februar 2012 eingereicht. ${ }^{45}$ Dort wurde ein weiterer Antrag vorgestellt, der zuvor nicht im LiquidFeedback behandelt worden war. Dieser warb ebenfalls für eine Klarnamenpflicht, setzte sich zusätzlich aber dafür ein, dass allen Gebietsversammlungen und Bezirksverbänden einschließlich ihrer Untergliederungen die Möglichkeit gegeben werden sollte, anstelle des Landessystems von LiquidFeedback eigene Plattformen und individuelle Regelungen nutzen zu dürfen. ${ }^{46}$ In der direkten Abstimmung setzte sich der neue Antrag gegen den ursprünglichen, der in LiquidFeedback mit Mehrheit angenommen worden war, durch. Der LiquidFeedback-Antrag kam somit nicht in die Endabstimmung. Für den neuen Antrag stimmten schließlich 107 Mitglieder (56,9 Prozent), 81 Personen (43,1 Prozent) stimmten dagegen. Folglich war auch dieser Antrag durchgefallen, da er nach den Reglements eine Zwei-Drittel-Mehrheit hätte erreichen müssen. ${ }^{47}$ Die unterschiedlichen Abstimmungsergebnisse wurden von dem interviewten Vertreter des Landesvorstandes der Piratenpartei damit begründet, dass die Stimmung auf einer Landesmitgliederversammlung durch besonders prägnante und aufrüttelnde Redebeiträge und dadurch auch die Ergebnisse beeinflusst werden könnten. Nach dem unbefriedigenden Ausgang des Abstimmungsverfahrens diskutierte der Landesvorstand auf seiner nächsten Sitzung am 4. März 2012 erneut über die Klarnamenpflicht. Dort wurde der Vorschlag, zwei LiquidFeedback-Systeme parallel laufen zu lassen - eins mit Klarnamenpflicht und eins ohne - einstimmig abgelehnt. ${ }^{48}$

(b) Fraktion im Berliner Abgeordnetenhaus: Unterstützung einer gesetzlichen Frauenquote

Die Nutzung von Liquid Democracy ist auch in der Satzung der Fraktion der Piratenpartei

44 Vgl. dass., Initiative \#1484, https://lqpp.de/be/initiative/show/1484.html (Abruf am 21. Mai 2014).

45 Vgl. Piratenwiki, Satzungsänderungsantrag 003 für die Landesmitgliederversammlung 2012.1, http://wiki.piratenpartei.de/BE:Antragskommission/LMV_2012.1/Antragsportal/Satzungs\% C3\% A4nderungsantrag_-_003 (Abruf am 21. Mai 2014).

46 Vgl. dass., Satzungsänderungsantrag 011 für die Landesmitgliederversammlung 2012.1, http:// wiki.piratenpartei.de/BE:Antragskommission/LMV_2012.1/Antragsportal/Satzungs\%C3\% A4nderungsantrag_-_011 (Abruf am 21. Mai 2014).

47 Vgl. dass., Protokoll der Landesmitgliederversammlung 2012.1, http://wiki.piratenpartei.de/ BE:Parteitag/2012.1/Protokoll (Abruf am 21. Mai 2014).

48 Vgl. dass., Protokoll Vorstandssitzung Landesverband Berlin vom 4. März 2012, http://wiki.piratenpartei.de/BE:2012-03-04_-_Protokoll_Vorstandssitzung_Landesverband_Berlin (Abruf am 21. Mai 2014). 
im Berliner Abgeordnetenhaus verankert. ${ }^{49}$ In $\$ 11$ wird festgelegt, dass die Fraktion zur Willensbildung eine über das Internet laufende, geeignete Software nutzt. Jedoch macht $\$ 13$ zum Abstimmungsverhalten in Plenar- und Ausschusssitzungen deutlich, dass die Fraktionsmitglieder in der Wahrnehmung ihres freien Mandates nicht an Mehrheitsentscheidungen oder Weisungen gebunden sind.

Anfang Januar 2012 wurden im Themenbereich „Wirtschaft und Soziales“ vier Initiativen zum Thema \#654 im Schnellverfahren eingestellt. ${ }^{50}$ Alle beschäftigten sich mit einer möglichen Unterstützung der so genannten Berliner Erklärung durch die Piratenfraktion des Berliner Abgeordnetenhauses. Dabei handelt es sich um ein überparteiliches Bündnis, das die Forderung stellt, mindestens 30 Prozent der Aufsichtsratssitze börsennotierter Unternehmen mit Frauen zu besetzen. Das Bündnis wurde von weiblichen Abgeordneten aus dem Bundestag sowie führenden Frauenverbänden ins Leben gerufen. Wie aus einem Eintrag im Blog von Simon Kowalewski ${ }^{1}$, Abgeordneter und frauenpolitischer Sprecher der Berliner Piratenfraktion, hervorgeht, wurden drei der vier Initiativen von ihm eingestellt. Zum Hintergrund erklärte er, dass die frauenpolitische Sprecherin der CDU, Katrin Vogel, ihn am 8. Januar 2012 angeschrieben und gefragt habe, ob die Fraktion der Piratenpartei bereit wäre, eine gemeinsame Pressemitteilung zur Unterstützung der Berliner Erklärung mitzutragen. Da Kowalewski diese Entscheidung auf Grund des für ihn brisanten Themas nicht allein treffen wollte, habe er sich zur Nutzung von LiquidFeedback entschlossen, um die Meinung der Basis einzuholen. Zu seinen drei Initiativen trat eine weitere des Abgeordneten Alexander Morlang hinzu.

(1) Die Ursprungsinitiative \#1407 trat für eine gemeinsame Pressemitteilung aller Fraktionen des Berliner Abgeordnetenhauses zur Unterstützung der Berliner Erklärung ein, in der die frauenpolitischen Sprecherinnen beziehungsweise Sprecher aller Fraktionen der Forderung nach einer Frauenquote von 30 Prozent in Aufsichtsräten Nachdruck verleihen sollten. ${ }^{52}$

(2) In der Initiative \#1408 wurde gefordert, dass die Piratenfraktion weder die gemeinsame Erklärung unterschreiben noch eine eigene Pressemitteilung zu dieser Thematik herausgeben solle. ${ }^{53}$

(3) Der Abgeordnete Alexander Morlang forderte in der Initiative \#1409, sich in der Frage Frauenquote in keiner Weise zu positionieren, da sich die Partei noch in einem Meinungsbildungsprozess zu dieser Thematik befinde und dieser Prozess noch nicht abgeschlossen sei. ${ }^{54}$

(4) Die Initiative \#1410 plädiert ebenfalls für eine Nichtunterstützung der überparteilichen Positionierung. Stattdessen solle die Fraktion die Berliner Erklärung aber durch eine eigene Pressemitteilung kommentieren. ${ }^{55}$

49 Vgl. Piratenfraktion im Abgeordnetenhaus von Berlin, Satzung der Piratenfraktion im Abgeordnetenhaus von Berlin, Fassung vom 22. Juni 2012, https://www.piratenfraktion-berlin.de/wpcontent/uploads/2012/02/120622-SATZUNG.pdf (Abruf am 21. Mai 2014).

50 Vgl. LiquidFeedback der Piratenpartei Deutschland Berlin, Thema \#654, https://lqpp.de/be/issue/show/654.html (Abruf am 21. Mai 2014).

51 Vgl. Simon Kowaleski, Die Piraten und die Berliner Erklärung, 11. Januar 2012, http://simonkowalewski.de/?p=5 (Abruf am 21. Mai 2014).

52 Vgl. LiquidFeedback der Piratenpartei Deutschland Berlin, Initiative \#1407, https://lqpp.de/be/ initiative/show/1407.html (Abruf am 21. Mai 2014).

53 Vgl. dass., Initiative \#1408, https://qpp.de/be/initiative/show/1408.html (Abruf am 21. Mai 2014).

54 Vgl. dass., Initiative \#1409, https://qpp.de/be/initiative/show/1409.html (Abruf am 21. Mai 2014).

55 Vgl. dass., Initiative \#1410, https://lqpp.de/be/initiative/show/1410.html (Abruf am 21. Mai 2014). 
Nachdem die Diskussion am 14. Januar 2012 abgeschlossen worden war, zeigte sich bei der Abstimmung folgendes Ergebnis: 117 Parteimitglieder hatten persönlich oder über eine Delegation teilgenommen. Was bei seinerzeit circa 2.500 Mitgliedern im Landesverband 4,7 Prozent entspricht. Mit einer Zustimmung von 91 Prozent belegte die Initiative \#1409, die sich gegen jegliche Positionierung zur Berliner Erklärung aussprach, deutlich den ersten Rang. Dennoch wurde am 31. Januar 2012 die geplante gemeinsame Pressemitteilung aller Fraktionen veröffentlicht, die von allen frauenpolitischen Sprecherinnen beziehungsweise Sprechern unterzeichnet wurde - auch von Simon Kowaleski im Namen der Fraktion der Piratenpartei. ${ }^{56}$ Von dem interviewten Vertreter der Fraktion wurde dies damit begründet, dass die Meinungsbilder der Basis in die Entscheidungsprozesse einflössen und einen hohen Stellenwert in der Meinungsbildung hätten, es jedoch vorkommen könne, dass die Fraktion aufgrund persönlicher Sichtweisen einzelner Abgeordneter eine konträre Position vertrete und diese auch umsetze.

\subsection{Die Piratenpartei in Nordrhein-Westfalen}

Der Landesverband Nordrhein-Westfalen wurde am 9. Juni 2007 gegründet. Nach Bayern ist er nach Angaben der Piratenpartei mit rund 5.900 Mitgliedern zweitgrößter Landesverband. Mit 7,8 Prozent zog die Partei bei der vorgezogenen Landtagswahl im Mai 2012 in den Landtag ein und stellt seitdem 20 Abgeordnete. Der Landesparteitag tagt mindestens einmal pro Jahr. An den Landesparteitagen in den Jahren 2011 und 2012 haben durchschnittlich 8,6 Prozent der Parteimitglieder teilgenommen (vgl. Tabelle 3).

\begin{tabular}{|l|c|c|c|}
\hline Tabelle 3: Teilnehmerzahlen der Landesparteitage der Piratenpartei Nordrhein-Westfalen \\
\hline Landesparteitag & Anzahl Teilnehmer & Anzahl Parteimitglieder & Anteil (in Prozent) \\
\hline 2012.3 (Dortmund) & 550 & 6.329 & 8,7 \\
2012.2 (Dortmund) & 450 & 5.410 & 8,3 \\
2012.1 (Münster) & 398 & 4.313 & 9,2 \\
2011.2 (Soest) & 270 & 3.145 & 8,6 \\
2011.1 (Gelsenkirchen) & 165 & 2.076 & 7,9 \\
\hline \multicolumn{4}{|l|}{ Durchschnitt } \\
\hline \multicolumn{4}{|l|}{ Quelle: Eigene Darstellung und Berechnung auf Basis von Angaben des Vorstandes der Piratenpartei } \\
\hline
\end{tabular}

(a) Landesverband Nordrhein-Westfalen: Positionierung in der Beschneidungsdebatte

Auch die Piraten in Nordrhein-Westfalen nutzen die Internetplattform LiquidFeedback zur Umsetzung des Liquid Democracy-Ansatzes. Auf ihrem Landesparteitag im April 2014 haben die Piraten aus Nordrhein-Westfalen zudem die Einführung einer ständigen Mitgliederversammlung im Internet beschlossen. Diese kann Stellungnahmen, Positionspapiere, Anfragen von Fraktionen sowie Anträge zu Programmen verbindlich beschließen; in ande-

56 Vgl. Katrin Vogel, Berliner Erklärung - Mitmachen!, 31. Januar 2012, http://www.katrinvogel.de/ index.php?ka=1\&ska=1\&idn=263 (Abruf am 21. Mai 2014). 
ren Bereichen (zum Beispiel bei Anträgen zur Satzung und Finanzordnung sowie zur Auflösung oder Verschmelzung mit anderen Parteien) kann sie lediglich Empfehlungen abgeben. 57

Im Schnellverfahren wurde Ende Juli 2012 im Bereich „Bürgerrechte, Datenschutz und Sicherheitspolitik“ das Thema \#116 im LiquidFeedback eingestellt. ${ }^{58}$ Dabei ging es um die Frage, ob der Landesvorstand einen offenen Brief des Medizinprofessors Matthias Franz unterzeichnen solle, der sich an die Bundesregierung und den Bundestag richtete und sich gegen die Beschneidung von Jungen aus religiösen Gründen aussprach. ${ }^{59}$ Das LiquidFeedback-Thema bestand aus zwei Initiativen:

(1) In der Initiative \#157 wurde der Landesvorstand aufgefordert, den offenen Brief zu unterschreiben. Die Initiatoren des Vorschlags begründeten ihre Aufforderung damit, dass man sich als Bürgerrechtspartei klar positionieren solle, wenn es um Gewaltanwendung gegen Kinder geht. ${ }^{60}$

(2) Die Initiative \#158 wandte sich gegen eine Unterzeichnung des offenen Briefes, mit der Begründung, dass selbst im Falle eines gesetzlichen Verbots Beschneidungen weiterhin durchgeführt würden, nur unter schlechteren Bedingungen. Daher wurde mehr Aufklärung in der Thematik statt eines Verbots gefordert. Zudem gestalte es sich schwierig, ohne einen Parteitagsbeschluss eine Position zu ergreifen. ${ }^{61}$

Die Initiative \#160 plädierte für einen besonneneren Umgang mit der Thematik und verlangte, weitere Expertenmeinungen hinzuzuziehen, um alle Seiten zu Wort kommen zu lassen. ${ }^{62}$ Der Vorschlag verfehlte jedoch im Vorfeld der Abstimmungen das nötige Quorum von zehn Prozent und wurde somit nicht zur Abstimmung zugelassen. Die Abstimmung über die beiden zugelassenen Initiativen wurde am 26. Juli 2012 abgeschlossen, dabei wurden die Stimmen von 70 Parteimitgliedern abgegeben. Bei 6.278 Mitgliedern im gesamten Landesverband zum Zeitpunkt der Abstimmung entspricht dies einer Teilnahmequote von nur 1,1 Prozent. 85 Prozent sprachen sich für die Initiative \#157 und somit für eine Unterzeichnung des offenen Briefs durch den Landesvorstand aus. Das Meinungsbild der kleinen Anzahl der teilnehmenden Mitglieder war somit eindeutig.

Daraufhin wandte sich die Arbeitsgemeinschaft Öffentlichkeitsarbeit des Landesverbandes an den Vorstand mit dem Hinweis auf die Abstimmung und der Bitte, den Brief entsprechend zu unterstützen. Der Landesvorstand unterzeichnete ihn jedoch nicht. Die Begründung lautete, dass lediglich 70 Stimmen abgegeben worden seien, davon etwa die Hälfte delegierte Stimmen. Bei einem Landesverband von solch einer Größe könne der

57 Vgl. Piratenpartei Deutschland Landesverband Nordrhein-Westfalen, Satzung der Piratenpartei Deutschland Landesverband Nordrhein-Westfalen, Fassung 2014, http://www.piratenpartei-nrw. de/landesverband/satzung/ (Abruf am 31. Mai 2014).

58 Vgl. LiquidFeedback der Piratenpartei Deutschland Landesverband Nordrhein-Westfalen, Thema \#116, https://lqpp.de/nw/issue/show/116.html (Abruf am 21. Mai 2014).

59 Vgl. Matthias Franz, Offener Brief zur Beschneidung „Religionsfreiheit kann kein Freibrief für Gewalt sein“, in: FAZ.net vom 21. Juli 2012, http://www.faz.net/aktuell/politik/inland/offenerbrief-zur-beschneidung-religionsfreiheit-kann-kein-freibrief-fuer-gewalt-sein-1 1827590.html (Abruf am 21. Mai 2014).

$60 \mathrm{Vgl}$. LiquidFeedback der Piratenpartei Deutschland Landesverband Nordrhein-Westfalen, Initiative \#157, https://qpp.de/nw/initiative/show/157.html (Abruf am 21. Mai 2014.)

$61 \mathrm{Vgl}$. dass., Initiative \#158, https://qpp.de/nw/initiative/show/158.html (Abruf am 21. Mai 2014).

62 Vgl. dass., Initiative \#160, https://lqpp.de/nw/initiative/show/160.html (Abruf am 21. Mai 2014). 
Vorstand eine Abstimmung mit so geringer Beteiligung „nicht wirklich ernst nehmen“, wie der interviewte Vertreter des Landesvorstandes sagte. Zudem hätten bereits Gruppierungen den Brief unterstützt, mit denen die Partei nichts zu tun haben wolle. Außerdem sei das Thema innerhalb der Partei sehr kontrovers diskutiert worden, und es gebe in den eigenen Reihen sowohl Befürworter als auch Gegner religiöser Beschneidung.

\section{(b) Fraktion im Landtag Nordrhein-Westfalen: Novellierung des Nichtraucherschutzes}

Die Piratenfraktion im Landtag Nordrhein-Westfalen nutzt zur Willensbildung und Einholung von Meinungsbildern über das Internet gemäß $₫ 10$ und $\$ 11$ ihrer Satzung „eine geeignete Software“63. In ihrem Abstimmungsverhalten in Plenar- und Ausschusssitzungen, geregelt in $\$ 13$ der Satzung, sind die Fraktionsmitglieder in der Wahrnehmung ihres freien Mandates nicht an Mehrheitsentscheidungen oder Weisungen gebunden. Sie sind allerdings gehalten, unter anderem Entscheidungen, die im Liquid Democracy-System gefasst wurden, zu berücksichtigen. ${ }^{64}$

Am 26. Juni 2012 legte die rot-grüne Regierungskoalition in Nordrhein-Westfalen einen Änderungsentwurf zum Nichtraucherschutzgesetz vor. Er sah vor, für den Gaststättenbereich ein uneingeschränktes Rauchverbot auszusprechen. Zudem sollten die bestehenden Ausnahmen für Brauchtumsveranstaltungen, Festzelte und Raucherclubs aufgehoben werden. Ferner sollte der Nichtraucherschutz für Kinder und Jugendliche in Einzelbereichen verbessert werden, unter anderem durch ein Rauchverbot auf Kinderspielplätzen. ${ }^{65}$ Daraufhin wurde auf dem wenige Tage später stattfindenden Landesparteitag der Piratenpartei vom Landesarbeitskreis Drogenpolitik und der Arbeitsgemeinschaft Drogenpolitik auf Bundesebene ein Positionspapier zum Nichtraucherschutz zur Abstimmung vorgelegt. ${ }^{66} \mathrm{Im}$ Kern ging es darum, die geplante Novellierung des nordrhein-westfälischen Nichtraucherschutzgesetzes abzulehnen. Das totale Rauchverbot sei eine unzumutbare Bevormundung von Bürgern und Gastronomie. Im Laufe des Parteitages wurde der Antrag „mit breiter Mehrheit" angenommen ${ }^{67}$ und als offizielle Position der Piratenpartei Nordrhein-Westfalen veröffentlicht. ${ }^{68}$ Im Anschluss daran gab es jedoch Kritik seitens einiger Mitglieder, die sich

63 Piratenfraktion im Landtag Nordrhein-Westfalen, Satzung, o.O. 2012, http://www.piratenfraktion-nrw.de/satzung/ (Abruf am 5. Januar 2014).

64 Vgl. ebenda.

65 Vgl. Landtag Nordrhein-Westfalen, Gesetzentwurf der Landesregierung. Gesetz zur Änderung des Gesetzes zum Schutz von Nichtraucherinnen und Nichtrauchern in Nordrhein-Westfalen (Nichtraucherschutzgesetz NRW - NiSchG NRW), Drs. 16/125, Düsseldorf 2012, http://www.landtag. nrw.de/portal/WWW/dokumentenarchiv/Dokument/MMD16-125.pdf (Abruf am 21. Mai 2014).

66 Vgl. Piratenwiki, Sonstige Anträge für den Landesparteitag 2012.3, http://wiki.piratenpartei.de/ NRW:Landesparteitag_2012.3/Antr\%C3\%A4ge/Sonstige_Antr\%C3\%A4ge (Abruf am 21. Mai 2014).

67 Dass., Protokoll Landesparteitag 2012.3, http://wiki.piratenpartei.de/NRW:Landespartei tag_2012.3/Protokoll/Sonntag\#Fortsetzung_ Positionspapier_Nichtraucherschutzgesetz (Abruf am 21. Mai 2014).

68 Vgl. Piratenpartei Deutschland Landesverband Nordrhein-Westfalen, Positionspapier: Position zur Novellierung des Nichtraucherschutzgesetz NRW, 2. Juli 2012, http://www.piratenparteinrw.de/2012/07/02/position-zur-novellierung-des-nichtraucherschutzgesetz-nrw/ (Abruf am 21. Mai 2014). 
in zwei offenen Briefen an die Landtagsfraktion richteten und diese aufforderten, den Gesetzesentwurf der Landesregierung zu unterstützen. ${ }^{69}$

Die Piratenfraktion entschloss sich jedoch, einen Änderungsantrag als Gegenentwurf einzubringen. Dazu wurden im LiquidFeedback des Landesverbands im Themenbereich „Umwelt, Gesundheit und Ernährung “ zwei Themen eingestellt, um die Meinung der Basis einzuholen und in den Änderungsantrag einzuarbeiten. Beide beinhalteten jeweils zwei konträre Initiativen zum Nichtraucherschutz. Thema \#169 behandelte die Frage nach einem möglichen Rauchverbot in Festzelten und auf so genannten Brauchtumsveranstaltungen ${ }^{70}$ :

(1) Initiative \#241 sprach sich dafür aus, die bestehende Ausnahmeregelung für Festzelte und Brauchtumsveranstaltungen im Nichtraucherschutzgesetz zu belassen. ${ }^{71}$

(2) Initiative \#242 forderte hingegen ein Rauchverbot. ${ }^{72}$

Die von außerhalb der Fraktion eingebrachte Initiative \#24373, die eine rein zahlenmäßige Abfrage im LiquidFeedback zu diesem Thema kritisierte, scheiterte bereits im Vorfeld am nötigen Quorum. Die Abstimmung über die beiden übrigen Initiativen endete am 29. Oktober 2012 und brachte folgendes Ergebnis: 63 Prozent der Teilnehmer sprachen sich für ein Rauchverbot in Festzelten und auf Brauchtumsveranstaltungen aus, 47 Prozent unterstützen die Initiative \#241 und somit das bestehende Gesetz. An der Abstimmung beteiligten sich 76 Parteimitglieder, was bei einer Mitgliederzahl von 6.367 Piraten im Landesverband einem Anteil von lediglich 1,2 Prozent entspricht.

Das Thema \#171 sollte Klarheit über die Meinung der Parteimitglieder bezüglich eines Rauchverbots auf Kinderspielplätzen bringen ${ }^{74}$, ebenfalls auf Grundlage zweier gegensätzlicher Initiativen:

(1) Initiative \#245 plädierte für ein Rauchverbot auf Kinderspielplätzen. ${ }^{75}$

(2) Initiative \#246 hingegen positionierte sich gegen ein Rauchverbot auf Kinderspielplätzen im Freien. ${ }^{76}$

Die Auszählung der Stimmen wurde am 30. Oktober 2012 mit dem Ergebnis abgeschlossen, dass die Initiative für das Rauchverbot 47 Prozent Unterstützer fand, die Initiative gegen das Verbot 58 Prozent. Somit sprach sich eine Mehrheit der an der Abstimmung beteiligten Parteimitglieder gegen ein Rauchverbot auf Kinderspielplätzen aus. Im Gegensatz zum Thema \#169 beteiligten sich diesmal nur 37 Parteimitglieder, was einem Anteil von 0,6 Prozent entspricht.

Am 27. November 2012 wurde schließlich der Änderungsantrag der Piratenfraktion zum Gesetzesvorschlag der Landesregierung eingereicht. Darin wandte sich die Fraktion gegen ein

69 Vgl. Piratenwiki, Offener Brief an die Landtagsfraktion der Piraten in NRW, http://wiki.piratenpartei.de/wiki/images/f/fa/Offener_Brief.pdf (Abruf am 21. Mai 2014); dass., Offener Brief an die Landtagsfraktion der Piraten in NRW zur Debatte über die Novellierung des Nichtraucherschutzgesetzes, http://wiki.piratenpartei.de/wiki/images/8/86/Offener_Brief_II.pdf (Abruf am 21. Mai 2014).

70 Vgl. LiquidFeedback der Piratenpartei Deutschland Landesverband Nordrhein-Westfalen, Thema \#169, https://lqpp.de/nw/issue/show/169.html (Abruf am 21. Mai 2014).

71 Vgl. dass., Initiative \#241, https://qpp.de/nw/initiative/show/241.html (Abruf am 21. Mai 2014).

72 Vgl. dass., Initiative \#242, https://lqpp.de/nw/initiative/show/242.html (Abruf am 21. Mai 2014).

73 Vgl. dass., Initiative \#243, https://lqpp.de/nw/initiative/show/243.html (Abruf am 21. Mai 2014).

74 Vgl. dass., Thema \#171, https://lqpp.de/nw/issue/show/171.html (Abruf am 21. Mai 2014).

75 Vgl. dass., Initiative \#245, https://lqpp.de/nw/initiative/show/245.html (Abruf am 21. Mai 2014).

76 Vgl. dass., Initiative \#246, https://lqpp.de/nw/initiative/show/246.html (Abruf am 21. Mai 2014). 
Rauchverbot auf Kinderspielplätzen. ${ }^{77}$ Zudem sollte die Ausnahmeregelung für Festzelte und Brauchtumsveranstaltungen beibehalten werden. ${ }^{78}$ Die Positionierung der Parteibasis aus dem LiquidFeedback-Thema \#171 - kein Rauchverbot auf Kinderspielplätzen - wurde also von der Fraktion in den Änderungsantrag eingearbeitet, während das Meinungsbild der Mitglieder aus dem Thema \#169 - für ein Rauchverbot in Festzelten und auf Brauchtumsveranstaltungen - übergangen wurde. Daraufhin habe es, wie der interviewte Vertreter der Landtagsfraktion berichtete, über die Mailingliste der AG Öffentlichkeitsarbeit heftige Diskussionen gegeben, die schließlich dazu führten, dass die Positionierung zum Rauchverbot in Festzelten und Brauchtumsveranstaltungen wieder aus dem Antrag zurückgezogen wurde.

In der abschließenden Abstimmung im Landtag am 29. November 2012 wurde der eingebrachte Änderungsantrag der Piratenpartei mit den Stimmen der Regierungskoalition gegen die Stimmen der Piraten und der FDP bei einer Enthaltung der CDU-Fraktion abgelehnt. ${ }^{79}$ Das Gesetz zur Änderung des Nichtraucherschutzgesetzes wurde daraufhin mehrheitlich verabschiedet. In der Abstimmung votierten 17 Abgeordnete der Piratenfraktion gegen das Gesetz, zwei enthielten sich, und einer fehlte entschuldigt. ${ }^{80}$

\section{Liquid Democracy in der Piratenpartei zwischen Anspruch und Wirklichkeit}

Sowohl in Berlin als auch in Nordrhein-Westfalen nutzt die Piratenpartei das Internet zur Umsetzung einer innerparteilichen Liquid Democracy. Dadurch sollen möglichst viele Parteimitglieder die Gelegenheit erhalten, in die Willensbildung und die Entscheidungsprozesse innerhalb der Partei einzugreifen. Gleichzeitig sollen durch Liquid Democracy die Entscheidungen von Landesvorstand und Fraktion an den Willen der Basis geknüpft werden. Als zentrales digitales Instrument wird dabei LiquidFeedback genutzt, das als wichtigstes Abstimmungstool fungiert. Die Analyse der Fallbeispiele zeigt jedoch eine erhebliche Diskrepanz zwischen Anspruch und Wirklichkeit in der praktischen Umsetzung innerhalb der Piratenpartei.

Auffällig ist zunächst der Vergleich zwischen den Partizipationsraten bei Abstimmungen im LiquidFeedback (vgl. Tabelle 4) und auf Landesparteitagen. An den beiden Landesmitgliederversammlungen der Piratenpartei Berlin haben sich 2012 im Schnitt 11,6 Prozent der Parteimitglieder beteiligt; 2011 waren es nach Schätzungen des Landesvorstandes jeweils zwischen 10 und 20 Prozent. Die beiden untersuchten Prozesse im LiquidFeedback

77 Vgl. Landtag Nordrhein-Westfalen, Änderungsantrag der Fraktion der Piraten zu der Beschlussempfehlung und dem Bericht des Ausschusses für Arbeit, Gesundheit und Soziales - Drs. 16/1493 - zum Gesetz zur Änderung des Nichtraucherschutzgesetzes NRW (Drs. 16/125), Drs. 16/1549, Düsseldorf 2012, http://www.landtag.nrw.de/portal/WWW/dokumentenarchiv/Dokument/MMD16-1549.pdf (Abruf am 21. Mai 2014).

78 Vgl. Landtag Nordrhein-Westfalen, Änderungsantrag der Fraktion der Piraten zu der Beschlussempfehlung und dem Bericht des Ausschusses für Arbeit, Gesundheit und Soziales - Drs. 16/1493 - zum Gesetz zur Änderung des Nichtraucherschutzgesetzes NRW (Drs. 16/125), Drs. 16/1550, Düsseldorf 2012, http://www.landtag.nrw.de/portal/WWW/dokumentenarchiv/Dokument/MMD16-1550.pdf (Abruf am 21. Mai 2014).

79 Vgl. Landtag Nordrhein-Westfalen, Plenarprotokoll 16/15 vom 29. November 2012, Düsseldorf 2012, S. 1038, http://www.landtag.nrw.de/portal/WWW/dokumentenarchiv/Dokument/MMP 16-15.pdf (Abruf am 21. Mai 2014).

80 Vgl. ebenda, S. $1075 \mathrm{ff}$. 


\begin{tabular}{|l|c|c|c|c|}
\hline \multicolumn{2}{|c|}{ Tabelle 4: Übersicht über die Beteiligung im LiquidFeedback } \\
\hline \multicolumn{2}{|c|}{ LiquidFeedback-Thema } & Mitgliederzahl & abgegebene Stimmen & Anteil (in Prozent) \\
\hline \multirow{2}{*}{ Berlin } & Thema \#657 & 2.500 & 117 & 4,7 \\
& Thema \#663 & 2.700 & 137 & 5,1 \\
\hline \multirow{2}{*}{ Nordrhein- } & Thema \#116 & 6.278 & 70 & 1,1 \\
Westfalen & Thema \#169 & 6.367 & 76 & 1,1 \\
\multicolumn{4}{|l|}{ Quelle: Eigene Darstellung. } \\
Thema \#171 & 6.367 & 37 & 0,6 \\
\hline
\end{tabular}

kamen nur auf eine Beteiligungsrate von 4,7 beziehungsweise 5,1 Prozent. Noch gravierendere Unterschiede in der Beteiligung liefern die Fallbeispiele aus Nordrhein-Westfalen. An den Landesparteitagen in den Jahren 2011 und 2012 nahmen durchschnittlich 8,6 Prozent der Parteimitglieder teil. Im LiquidFeedback reichte es bei beiden Fallbeispielen jedoch nur zu Beteiligungsquoten zwischen 0,6 und 1,2 Prozent.

In Berlin, so der interviewte Vertreter des Landesverbandes, sei man dennoch mit der Partizipation der Parteimitglieder zufrieden, wenn auch mehr Beteiligung - sowohl online als auch offline - wünschenswert sei. Der Interviewpartner aus der Fraktion ergänzte, dass aufgrund der Möglichkeit der Stimmendelegation eine aktive Beteiligung nicht immer nötig sei, da die Stimmen dennoch repräsentiert würden. In Nordrhein-Westfalen bezeichnete der Vertreter des Landesverbandes das Beteiligungsniveau im LiquidFeeback jedoch als „sehr schlecht"; es sei „noch Luft nach oben“ vorhanden, wie der interviewte Vertreter der Fraktion im Landtag von Nordrhein-Westfalen ebenfalls unterstrich. Als möglicher Grund für das mangelnde Interesse an LiquidFeedback wurde angeführt, dass sich einige Parteimitglieder auf das Bezahlen von Beiträgen beschränken und sich bewusst nicht an Entscheidungsprozessen beteiligen wollten. Daher lasse sich die Beteiligungsquote nur sehr schwer bewerten, denn einige Mitglieder seien gern „stilles Mitglied“. Aufschlussreich ist auch die Einschätzung des interviewten Landesvorsitzenden aus Nordrhein-Westfalen, dass den Debatten im Internet manchmal der ,soziale Charakter" fehle und sich die Parteimitglieder hin und wieder auch von Angesicht zu Angesicht gegenüberstehen müssten. Partizipation könne „nicht nur digital stattfinden“.

Mit Blick auf die Ergebnisse der vier Fallbeispiele kann die erste Annahme - dass durch die niedrigschwelligen Hürden digitaler Partizipation sich mehr Mitglieder der Piratenpartei an innerparteilichen Entscheidungen im Internet beteiligen als auf den Parteitagen nicht bestätigt werden.

Die Abstimmungsergebnisse im LiquidFeedback hatten länder- und institutionsübergreifend zudem wenig Einfluss auf die letztendlichen Entscheidungen. In der Debatte um die Klarnamenpflicht im Landesverband Berlin wurde das Meinungsbild der Parteibasis zugunsten einer Einführung der Klarnamenpflicht durch einen kurzfristig auf der Landesmitgliederversammlung eingebrachten Antrag überstimmt. Der Landesvorstand orientierte sich daraufhin an diesem Ergebnis und nicht an dem der Online-Abstimmung. In der Debatte um die Unterstützung der Berliner Erklärung (Einführung einer gesetzlichen Frauenquote in Aufsichtsräten) entschied sich die Berliner Piratenfraktion ebenfalls gegen das Ergebnis der LiquidFeedback-Abstimmung. Ähnliche Ergebnisse liefert die Analyse der exemplarischen Entscheidungsprozesse bei der Piratenpartei und -fraktion in NordrheinWestfalen. Die Forderung der Parteibasis an den Landesvorstand, einen offenen Brief zur 
Ablehnung religiöser Beschneidungen zu unterzeichnen, wurde trotz eines positiven Meinungsbildes im LiquidFeedback nicht umgesetzt. Etwas anders gestaltete sich die Lage in der Diskussion um die Novellierung des Nichtraucherschutzgesetzes in Nordrhein-Westfalen. Hier wurde von der Fraktion im LiquidFeedback die Meinung der Mitglieder zur Gestaltung eines Änderungsantrages eingeholt und - nach einigen Interventionen seitens der Parteibasis - in den Antrag eingebaut. Zusammenfassend lässt sich vor diesem Hintergrund feststellen, dass sich auch die zweite Annahme - dass sich die Entscheidungsträger der Piratenpartei an die Meinungsbilder der Parteibasis halten, die durch die Anwendung von Liquid Democracy entstehen - in den untersuchten Fallbeispielen im Wesentlichen nicht bestätigt.

Die Interviewpartner der Piratenpartei verteidigten dennoch die praktizierten Formen der digitalen Mitgliederpartizipation. Es sei ihre Hauptaufgabe als Landes- beziehungsweise Fraktionsvorstand, eine moderierende, vermittelnde und verwaltende Funktion nach innen zu übernehmen. Die Entscheidungsprozesse verliefen laut Aussage des interviewten Landesvorsitzenden aus Berlin nach wie vor „bottom-up“. Der interviewte Fraktionsvorsitzende und der Landesvorsitzende aus Nordrhein-Westfalen betonten, dass man versuche, „möglichst viele Menschen am politischen Willensbildungsprozess zu beteiligen“ beziehungsweise die Meinung der Basis im Hinterkopf zu behalten. Die Einholung von Meinungsbildern über das Internet sei für viele Parteimitglieder „Gesetz“, und dieser basisdemokratische Aspekt sei bei vielen Piraten verinnerlicht. Dennoch werden die LiquidFeedback-Abstimmungen von allen Gesprächspartnern als nicht verbindlich angesehen, da die Mitgliederversammlungen gemäß Satzung einen höheren Stellenwert hätten und LiquidFeedback noch nicht institutionalisiert sei. Für die sehr geringe Beteiligung im LiquidFeedback führten die beiden Interviewpartner aus Nordrhein-Westfalen als Grund den nicht-verbindlichen Charakter des Tools an. Die Anwendung des Internets als Partizipationsplattform sei daher eine „Laborsituation“, wodurch „sich eine Beteiligungsdemokratie nicht realisieren“ lasse, so der Interviewpartner aus der Fraktion in Nordrhein-Westfalen. Die Plattform wird ihm zufolge vielmehr als „Ausarbeitungs- und Verfeinerungstool für Anträge“ betrachtet.

Der Anspruch, den die Piratenpartei im Hinblick auf die innerparteiliche Beteiligungsdemokratie durch die Nutzung von LiquidFeedback erhebt, wird insofern kaum eingelöst. Nicht ohne Grund gibt es innerhalb der Partei - sowohl auf Bundes- als auch auf den verschiedenen Landesebenen - andauernde Diskussionen über die zukünftige Gestaltung innerparteilicher Entscheidungsstrukturen. Dabei geht es vor allem um die Implementierung von institutionalisierten, rechtlich zulässigen und verbindlichen Abstimmungen über das Internet - die bereits erwähnten ständigen Mitgliederversammlungen. Angesichts der Komplexität und des erheblichen Zeitaufwandes, der - wie die Fallbeispiele der Untersuchung zeigen - mit der digitalen Mitgliederpartizipation über LiquidFeedback verbunden ist, ist es jedoch höchst unwahrscheinlich, dass es gelingt, solche verbindlichen Entscheidungsprozesse mit entsprechend hoher Beteiligung zu realisieren. Hinzu kommt die seit einigen Monaten andauernde Krise der Partei, die in dem bescheidenen Ergebnis bei der Bundestagswahl 2013 gipfelte. Sollten die Piraten trotz des aktuellen Abwärtstrends in der Lage sein, sich wieder zu stabilisieren, müssten sie die vielen Schwierigkeiten des Konzepts der Liquid Democracy überwinden, um daraus doch noch ein geeignetes Instrument für die innerparteiliche Demokratie und den Dialog mit der Gesellschaft zu schaffen. 